\title{
Insulin-Injection-related Needle-stick Injuries Among Clinical Nurses at a Tertiary-Care Hospital in China
}

\author{
Zhiqi Luo ${ }^{1}$, Na Li ${ }^{1}$, Jiewei Huang, ${ }^{2, *}$, Xueyan Liu ${ }^{3, ~ *, ~ P e i r u ~ Z h o u ~}{ }^{2}$ \\ ${ }^{1}$ School of Nursing, Jinan University, Guangzhou, China \\ ${ }^{2}$ Department of Nursing, The First Affiliated Hospital of Jinan University, Guangzhou, China \\ ${ }^{3}$ Endocrinology, The First Affiliated Hospital of Jinan University, Guangzhou, China
}

Email address:

luozhiqi1995@126.com (Zhiqi Luo),linazjinan@stu2018.jnu.edu.cn (Na Li),dmapn@126.com (Jiewei Huang),yhlu@163.com (Xueyan Liu) ${ }^{*}$ Corresponding author

\section{To cite this article:}

Zhiqi Luo, Na Li, Jiewei Huang, Xueyan Liu, Peiru Zhou. Insulin-injection-related Needle-stick Injuries Among Clinical Nurses at a Tertiary-care Hospital in China. American Journal of Nursing Science. Vol. 10, No. 1, 2021, pp. 17-23. doi: 10.11648/j.ajns.20211001.14

Received: December 26, 2020; Accepted: January 6, 2021; Published: January 12, 2021

\begin{abstract}
Needle-stick injuries (NSIs) are the main type of occupational injury experienced by health-care workers worldwide. They are widely reported in the literatures, but there have been few studies of insulin-injection-related NSIs. This study aimed to determine the prevalence of and risk factors for insulin-injection-related NSIs among clinical nurses working at a Chinese tertiary-care hospital. Methods: We used a questionnaire to investigate the incidence of and risk factors for insulin injection-related NSIs in a tertiary-care hospital in Guangzhou, China. The study involved 576 nurses from various departments, including endocrinology, internal medicine other than endocrinology, surgery, and obstetrics and gynecology. Results: Approximately half $(54 \%)$ of the participants reported having $>5$-years of experience in clinical nursing, and $66.3 \%$ reported that they had received training on injection safety at work. While $16.0 \%$ of the nurses had experienced an insulin-injection-related NSI before, $58.7 \%$ of them did not report it to the relevant hospital department. When NSIs occurred, $69.6 \%$ of nurses were not wearing gloves, and $75 \%$ of the needles had been contaminated by patients. The largest proportion (34.8\%) of the NSIs occurred when the needle cap was being re-attached after an injection. Multivariate logistic analysis showed that participation in training (odds ratio $[O R]=0.605)$ and being a diabetes specialist nurse $(\mathrm{OR}=1.814)$ were independent factors related to the incidence of insulin-injection-related NSI. Conclusion: Insulin-injection-related NSIs are common among clinical nurses. Hospital management departments need to improve their training of nurses in preventing and handling NSIs, provide appropriate safety equipment, and implement simpler procedures for reporting NSIs.
\end{abstract}

Keywords: Clinical Nurse, Insulin-injection-related Needle Stick Injuries, Risk Factors, China

\section{Introduction}

The global prevalence of diabetes in increasing, with the number of diabetes patients worldwide reaching 463 million in 2019, and this is predicted to increase to 700 million in 2045. China has the largest number of diabetics in the world, with 116.4 million adult diabetic patients [1]; their prevalence increased from $0.67 \%$ in 1980 to $10.9 \%$ in 2013 [2-5]. Diabetes mellitus covers a group of metabolic diseases characterized by hyperglycemia that result from deficient of insulin secretion, insulin effects, or both mechanisms [6]. Many patients with diabetes require insulin therapy, and so the rapid increase in the prevalence of diabetes in China has resulted in the rate of insulin use also increasing.

The incidence of needle-stick injury (NSI) is at least as high among nursing staff performing insulin injections as it is in other medical departments and wards. A NSI refers to a skin injury caused by a sharp instrument such as a scalp needle, syringe needle, intravenous catheter, blood sampling needle, or hypodermic needle [7]. A survey involving 14 European nations found that nearly one-third of nurses giving injections to diabetics in hospitals have experienced NSIs [9]. A very large survey of insulin-injection-related NSIs among hospital nurses in China found that $39.1 \%$ of nurses had experienced at least one NSI related to insulin injection [8].

A NSI caused by an insulin pen may transmit at least 30 types of life-threatening blood-borne pathogens, including 
hepatitis B virus (HBV), hepatitis $\mathrm{C}$ virus (HCV), and human immunodeficiency virus (HIV) [9]. NSIs caused by insulin injections reportedly accounted for $3.2 \%$ and $0.9 \%$ of cases of nurses being infected with $\mathrm{HBV}$ and $\mathrm{HCV}$, respectively [8]. Insulin-injection-related NSIs will not only cause physical injury and have an emotional impact on the medical staff, but they are also associated with significant direct, indirect, and intangible cost [10], that further results in economic losses at the society and country levels $[11,12]$.

Currently there are about 86 million people infected with HBV in China, which accounts for the largest proportion (30\%) of the infected people worldwide [13]. In addition, in recent years an average of 1 million new cases of hepatitis $B$ have occurred in China [14] and the incidence of HIVs is also on the rise [15]. Therefore, NSIs caused by insulin injections represent a serious occupational hazard for Chinese nurses. Despite this situation, there have been few investigations of the status of and risk factors for insulin-injection-related NSIs in Chinese nurses.

The purpose of this study was to understand the problems experienced by clinical nurses when they are performing insulin injections, including the incidence of insulin injection-related NSIs, and to identify the risk factors for these injuries.

\section{Material and Methods}

\subsection{Setting and Subjects}

This study was performed in a Chinese tertiary-care hospital in Guangdong, Province, China during July 2020, with convenience sampling method used to select 576 nurses as the research objects. The inclusion criteria were (1) registered nurses engaged in clinical nursing work and (2) providing informed consent and participating voluntarily. Nursing interns and nurses in administrative roles who were not involved in the direct care of patients were excluded.

\subsection{Measurements}

A questionnaire was prepared that included general demographic characteristics and insulin-injection-related NSI, and it was approved by the Diabetes Committee of the Chinese Nursing Association.

\subsection{Data Collection}

Data collection began in July 2020, with the investigators using an online survey via a unified training platform (https://www.wenjuan.com/). Subjects meeting the eligibility criteria were sent an electronic questionnaire survey, the respondents anonymously completed the questionnaires online, and the online survey platform automatically collected data while, following the principle of informed consent. The questionnaire data collection process was completed within 1 week. All of the 576 recovered questionnaires were valid, giving an effective recovery rate of $100 \%$.

The first part of the questionnaire collected demographic data. The second part collected information on whether the participants had experienced insulin injection-related NSIs and the characteristics of the NSIs, including the device and activity that caused the NSI, the degree of contamination of the sharp instrument, the protective measures taken, and the degree of injury.

\subsection{Statistical Analysis}

After preprocessing the original data, SPSS (version25.0, IBM, NY, USA) was used to establish a database for statistical analysis. Descriptive statistical analysis was used to summarize the study variables, and the chi-square test was used to assess the associations between insulin-injection-related NSI and selected demographic factors. In addition, variables that were statistically significant in the chi-square test were screened into a logistic regression model for further analysis while controlling for confounding factors. The dependent variable in the logistic regression analysis was insulin-injection-related NSI (yes/no), while the independent variables were being a diabetes specialist nurse (yes/no), receiving training (yes/no), type of working department, and number of department beds. The criterion for statistical significance was set as $p<0.05$.

\section{Results}

\subsection{Demographic Characteristics of Participants}

The 576 nurses who participated in the survey all answered the survey questions completely. Demographic factors, job characteristics, and previous participation in training courses were all included in the further analysis to identify the factors that affect the occurrence of NSIs. General information and the results obtained in the single-factor analysis are presented in Table 1. The respondents were from department of endocrinology $(2.4 \%)$, internal medicine other than endocrinology $(29.2 \%)$, surgery $(27.3 \%)$, obstetrics and gynecology $(13.2 \%)$, and other departments $(28.0 \%)$. Approximately half (54\%) of the nurses reported to having $>5$-years of nursing experience, $57.6 \%$ had a job title of senior nurse, and $66.3 \%$ reported that they had received training on injection safety at work.

\subsection{General Condition of Needle-stick Injury}

The respondents included $92(16 \%)$ who had experienced an insulin-injection-related NSI. The rate of NSIs was significantly higher in certified diabetes specialist nurses $(23.8 \%)$ than among other nurses $(14.7 \%)$, and significantly lower in nurses who had participated in training for preventing NSIs $(13.6 \%)$ than in those who had not received such training $(20.6 \%)$, the differences were statistically significant $(p$ $<0.05)$.

The incidence of NSIs also varied between departments, being significantly higher in departments with $31-50$ beds ( $p$ $<0.05$ ); and in endocrinology departments than in departments of internal medicine (other than endocrinology), surgery, and obstetrics and gynecology $(p<0.05)$. 
Table 1. Comparison of insulin-injection-related NSIs according to selected demographics factors.

\begin{tabular}{|c|c|c|c|c|}
\hline Category & Data & Person with injury n (\%) & $\overline{X^{2}}$ & $p$ value \\
\hline Total & 576 & $92(16 \%)$ & & \\
\hline \multicolumn{5}{|l|}{ Work experience } \\
\hline in 2 years & $86(14.9 \%)$ & $7(8.1 \%)$ & 4.869 & 0.182 \\
\hline $3-5$ years & $179(31.1 \%)$ & $31(17.3 \%)$ & & \\
\hline $6-10$ years & $159(27.6 \%)$ & $27(17.0 \%)$ & & \\
\hline$>10$ years & $152(26.4 \%)$ & $27(17.8 \%)$ & & \\
\hline \multicolumn{5}{|l|}{ Diabetes specialist nurse } \\
\hline No & $496(86.1 \%)$ & $73(14.7 \%)$ & 4.187 & 0.041 \\
\hline Yes & $80(13.9 \%)$ & $19(23.8 \%)$ & & \\
\hline \multicolumn{5}{|l|}{ Bed's number } \\
\hline $1-30$ & $110(19.1 \%)$ & $8(7.3 \%)$ & 14.206 & 0.003 \\
\hline $31-50$ & $190(33.0 \%)$ & $44(23.2 \%)$ & & \\
\hline $51-70$ & $162(28.1 \%)$ & $22(13.6 \%)$ & & \\
\hline$>70$ & $114(19.8 \%)$ & $18(15.8 \%)$ & & \\
\hline \multicolumn{5}{|l|}{ Job title } \\
\hline Nurse & $194(33.7 \%)$ & $25(12.9 \%)$ & 3.425 & 0.331 \\
\hline Senior nurse & $332(57.6 \%)$ & $58(17.5 \%)$ & & \\
\hline Nurse-in-charge & $45(7.8 \%)$ & $9(20.0 \%)$ & & \\
\hline Deputy chief nurses & $5(0.9 \%)$ & $0(0.0 \%)$ & & \\
\hline \multicolumn{5}{|l|}{ Prior NSIs prevention training education } \\
\hline No & $194(33.7 \%)$ & $40(20.6 \%)$ & 4.705 & 0.030 \\
\hline Yes & $382(66.3 \%)$ & $52(13.6 \%)$ & & \\
\hline \multicolumn{5}{|l|}{ Work department } \\
\hline Endocrinology & $14(2.4 \%)$ & $5(35.7 \%)$ & 9.705 & 0.046 \\
\hline Internal medicine (Other than endocrinology) & $168(29.2 \%)$ & $29(17.3 \%)$ & & \\
\hline Surgery & $157(27.3 \%)$ & $31(19.7 \%)$ & & \\
\hline Obstetrics/gynecology & $76(13.2 \%)$ & $8(10.5 \%)$ & & \\
\hline Others department & $161(28.0 \%)$ & $19(11.8 \%)$ & & \\
\hline
\end{tabular}

Multiple insulin-injection-related NSIs had been experienced by $42.2 \%$ of the clinical nurses. Among those who had experienced any NSIs, $60.9 \%$ had skin punctures and bleeding, $69.6 \%$ were not wearing gloves at the time of their NSIs, and $75 \%$ of the needles had been contaminated by patients (Table 2).

Table 2. Specific situations of insulin-injection-related NSI $(n=92)$.

\begin{tabular}{|c|c|c|}
\hline Item & Number & Composition (\%) \\
\hline \multicolumn{3}{|l|}{ Times of insulin-injection-related NSIs } \\
\hline 1 Times & 52 & 57.8 \\
\hline 2 Times & 29 & 31.5 \\
\hline 3 Times & 6 & 6.5 \\
\hline$\geq 4$ Times & 5 & 5.4 \\
\hline \multicolumn{3}{|l|}{ Severity of injuries } \\
\hline Superficial (no or almost no bleeding) & 36 & 39.1 \\
\hline Moderate (skin puncture, a certain amount of bleeding) & 55 & 59.8 \\
\hline Severe (deep penetration/cutting, or significant bleeding) & 1 & 1.1 \\
\hline \multicolumn{3}{|l|}{ If the hand gets injured, whether the needle penetrates the hand } \\
\hline A pair of gloves & 23 & 25.0 \\
\hline Two pairs of gloves & 5 & 5.4 \\
\hline No gloves & 64 & 69.6 \\
\hline \multicolumn{3}{|l|}{ Contamination degree of sharp objects } \\
\hline Uncontaminated & 21 & 22.8 \\
\hline Unknown & 2 & 2.2 \\
\hline
\end{tabular}

\subsection{Risk Factors for NSI}

Multivariate logistic regression analysis was applied to the factors that were statistically significant in the single-factor analysis (Table 1), with the likelihood-ratio forward method used to screen the factors at a threshold of $\alpha=0.05$. The results are listed in Table 3. Participation in needle-stick knowledge training and being a diabetes specialist nurse were independent factors influencing insulin-injection-relate NSIs.

The risk of insulin-injection-related NSIs was lower in nurses who had not received hospital needlestick training (odds ratio $[\mathrm{OR}]=0.605,95 \%$ confidence interval $[\mathrm{CI}]=0.383,0.954)$, and higher in diabetes specialist nurses than in non-diabetes specialist nurses $(\mathrm{OR}=1.814$, $95 \% \mathrm{CI}=1.021,3.223)$. 
Table 3. Results from a logistics regression model of insulin-injection-related NSIs.

\begin{tabular}{lll}
\hline Variances & OR $\mathbf{( 9 5 \% C I )}$ & P value \\
\hline Prior NSIs prevention training & $0.605(0.383,0.954)$ & 0.031 \\
education & & \\
No & \\
Yes & $1.814(0.383,0.954)$ & 0.042 \\
Diabetes specialist nurse & \\
No & \\
Yes & \\
CI, confidence intervals. & \\
\hline
\end{tabular}

\subsection{Devices Involved and Times of Occurrence of NSIs}

Table 4 lists the types of devices that caused insulin-injection-related NSIs and the times when they occurred. Most NSIs had occurred when using traditional insulin pens without safety equipment, and the largest proportion (34.8\%) of the NSIs occurred when the needle cap was reattached after an injection.
Table 4. Devices involved and times of occurrence of NSIs.

\begin{tabular}{lll}
\hline Item & Num & $\begin{array}{l}\text { Composition } \\
(\%)\end{array}$ \\
\hline Types of equipment with NSIs & & \\
Traditional syringe needle (no safety device) & 20 & 21.7 \\
Traditional insulin pen needle (without safety device) & 70 & 76.1 \\
Insulin pump needle & 2 & 2.2 \\
Times of occurrence of NSIs & & \\
Before using the needle & 9 & 9.8 \\
Using the needle & 3 & 3.3 \\
Transfer instrument & 1 & 1.1 \\
When putting on the needle cap again & 32 & 34.8 \\
When putting items in the sharps box & 13 & 14.1 \\
After disposal & 8 & 8.7 \\
Patient holding contaminated needle & 6 & 6.5 \\
\hline
\end{tabular}

\subsection{Management After Occurrence of NSIs}

Most (58.7\%) of nurses with insulin-injection-related NSIs did not report the injury to the relevant hospital departments. The main reasons for not reporting the injury were "I think the incident has no health risk" $(58.7 \%)$ and "I was very busy at the time" (28.2\%) (Table 5).

Table 5. Reports of insulin-injection-related NSIs $(n=92)$.

\begin{tabular}{|c|c|c|c|}
\hline Item & Classification & Num & Composition (\%) \\
\hline \multirow[t]{2}{*}{ Report after NSIs } & Yes & 38 & 41.3 \\
\hline & No & 54 & 58.7 \\
\hline \multirow[t]{5}{*}{ Reasons for not reporting } & "I don't think the incident has health risks" & 54 & 58.7 \\
\hline & "I was very busy" & 26 & 28.2 \\
\hline & "I was so embarrassed" & 5 & 5.4 \\
\hline & "I think reporting may have a negative impact on my work/career" & 6 & 6.5 \\
\hline & "I don't want to know the result" & 1 & 1.1 \\
\hline
\end{tabular}

\section{Discussion}

\subsection{Analysis of the Occurrence of and Risk Factors for Insulin-injection-related NSIs}

Previous researches on NSIs have mostly focused on the overall NSI status of medical staff, and few studies have investigated the incidence of insulin-injection-related NSI and their influencing factors. In the present survey, $16.0 \%$ of nurses reported experiencing at least one insulin-injection-related NSI, which is lower than the rates found by Zhao et al. [16] (39.1\%), Dong et al. [8] (19.33\%), and Costigliola et al. [9] (32\%). Two large-scale surveys in China found that the overall incidence rates of NSIs among nurses were $7.8 \%$ [17] and $10.3 \%$ [17, 18], which are lower than the rate found in this study. These discrepancies in the incidence of NSIs among different studies may be related to factors such as the included hospitals and countries [19]; however, overall the results of the various studies show that there is a relatively high incidence of NSIs caused by insulin injection among clinical nurses [20]. Meanwhile, NSI is a transmission route for $\mathrm{HIV}, \mathrm{HBV}$, and $\mathrm{HCV}$, and it is reported that the prevalence of AIDS, hepatitis $B$, and hepatitis $C$ in diabetic patients is at least as high as those in healthy people and patients with other diseases [20-23]. Therefore, the risk of blood-borne infections may be higher in nurses who experience an insulin-injection-related NSI, which need to be addressed by both hospital managements and the actual nursing staff.

This study also found that the risk of insulin injection-related NSIs is affected by factors such as receiving training on preventing NSI, being a diabetes specialist nurse, departments type, and number of beds. However, the multivariate logistic regression analysis showed that receiving training and being a diabetes specialist nurse are independent factors influencing the occurrence of insulin-injection-related NSIs.

The risk of NSI was found to be lower in nurses who had received training, which is consistent with previous reports of training significantly reducing the incidence of NSIs among medical staff [20, 24-26]. Moreover, organizing training is cheaper and more feasible than providing safety devices and other NSI prevention measures, which makes it worth promoting [24, 27]. The present study found that, diabetes specialist nurses had a 1.8-fold higher risk of insulin-injection-related NSIs compared with non-specialist nurses. The core role of the diabetes specialist nurse is as a clinical caregiver [28, 29], and so ward nurses may need to perform more injection operations when they are looking after diabetic patients. At the same time, unlike foreign specialist 
nurses who need to complete regular assessments after obtaining certification, most domestic specialist nurses only need to pass a single assessment in China [30]. The insufficient follow-up management and guidance of specialist nurses may mean that the abilities of diabetes specialist nurses need to be further improved.

\subsection{What Happens When a NSI Occurs}

Medical staff should regard the blood and body fluids of patients as sources of infections of blood-borne infectious diseases such as HIV, HBV, and HCV. Moreover, the transmission risk of blood-borne pathogens is three times higher for deep damage than for surface damage [20]. Therefore, medical staff should wear gloves whenever they are performing procedures in which they may come into contact with the blood and body fluids of patients in order to prevent occupational exposure. In this study, $69.6 \%$ of nurses were not wearing gloves when they experienced a NSI, which is a higher proportion than in previous studies [31]. Moreover, nearly half of the nurses had experienced multiple stabbing wounds. Most nurses experience NSIs in which the skin is punctured and bleeding, and nurses are at a greater risk of blood-borne infections. Previous studies have shown that medical staff who wear gloves either only occasionally or never have a higher risk of NSIs than do those who wear gloves regularly [26]. Mischke et al. [32] demonstrated that the use of double-layer gloves can reduce the occurrence of NSIs without affecting their dexterity.

In the present study, $75 \%$ of the devices had been contaminated when an insulin-injection-related NSI occurred, which is similar to the findings of previous NSI surveys conducted in Korea [33] (60.6\%) and China [18] (77.5\%). More than two-thirds of insulin-injection-related NSIs experienced by clinical nurses involved contaminated sharps. There are more than 80 million patients with hepatitis $\mathrm{B}$ in China [34], and the incidence of hepatitis $C$ is 16 cases per 100,000 people, and the number of people infected with HIV exceeds 570,000 [35]. These three diseases are how major epidemics in China, and so Chinese nurses must be aware of their high risk of being infected by blood or body fluid pathogens on contaminated devices.

\subsection{Types of Syringes That Cause Insulin-injection-related NSIs and the Times When They Occur}

Most of the NSIs in this study occurred when using traditional insulin pens without safety equipment. Many previous studies [20, 24, 36, 37] have demonstrated how important safety equipment is to preventing NSIs. EU legislation enacted in 2013 stipulated that medical workers must use safety equipment when performing dangerous injections, including for diabetic patients [20]. The largest proportion $(34.8 \%)$ of the NSIs in the present study occurred when the needle cap was being re-attached after an injection. Other studies have shown that recapping needle is an important risk factor for NSIs [38, 39]. The safe injection guidelines of the World Health Organization [40] forbid the disassembly or retraction of the needle after an injection, and state that the needle and syringe should be integrated immediately and placed in a sharps box.

It had been reported [20] that the provision of adequate safety equipment can effectively reduce the occurrence of NSIs caused by trocars and other devices, while cost-benefit analysis showed that the cost savings of using safety equipment to reduce injuries from sharp objects can offset the increased hospital costs associated with using such equipment [16]. Therefore, hospital managers should provide nurses with safety equipment for use with insulin injection tools.

\subsection{Reporting After a NSI}

In this study, after a NSI occurred, most of the nurses failed to report the injury to the relevant department of the hospital. The main reason for not reporting the injury was "I think the incident has no health risk" ( $58.7 \%)$, followed by "I was very busy at the time" $(28.2 \%)$, which is similar to the results of Dong et al. [8], Joukar et al. [41], and Sabermoghaddam et al. [42]. Nurses may think that an insulin injection device will not be contaminated by blood, which will reduce their vigilance. However, it has been shown previously that blood attached to a used injection device is sometimes not visible. Only small amounts of infected blood are needed to transmit highly contagious viruses such as HBV, which can be present even when a device is not used specifically for drawing blood or obtaining vascular access [20].

Previous research results demonstrate that training can improve the above-mentioned problems such as not reporting NSIs, not wearing protective gloves, and retracting used needles [27]. Hospital managers need to provide appropriate training programs, regularly update staff with knowledge about NSIs, improve the general safety awareness of medical staff, and establish a rapid and effective reporting system. After a NSI occurs, managers need to strengthen the guidance and support to nurses, and arrange for special personnel to help with this.

\section{Conclusions}

Clinical nurses experience a high incidence of insulin-injection-related NSIs, and most of them fail to report such events. Such injuries should not be ignored. The occurrence rate of NSIs is significantly affected by factors such as training, department type, number of beds, and being a diabetes specialist nurse, with participation in NSI knowledge training and being a diabetes specialist nurse being the most important independent factors. Hospital managers need to strengthen training on NSI prevention and provide safety equipment, provide standardized insulin injection training for nurses based on the latest guidelines, and comprehensively improve the awareness that clinical nurses have of injection guidelines and the adverse effects of NSIs.

The main limitations of this study were that the survey subjects only included clinical nurses in a single tertiary-care hospital in Guangdong Province, and so the scope of the survey needs to be expanded in the future. Moreover, the survey subjects used self-evaluations to complete the 
questionnaire, and so future studies should attempt to obtain more objective research results.

\section{Author Contributions}

Zhiqi Luo and $\mathrm{Na} \mathrm{Li} \mathrm{are} \mathrm{co-first} \mathrm{authors.}$

\section{Conflict of Interest}

All the authors do not have any possible conflicts of interest.

\section{Acknowledgements}

The authors gratefully acknowledge the financial supports by the Natural Science Foundation of Guangdong Province of China under Grant numbers 2016B010108008.

\section{References}

[1] Saeedi P, Petersohn I, Salpea P, et al. Global and regional diabetes prevalence estimates for 2019 and projections for 2030 and 2045: Results from the International Diabetes Federation Diabetes Atlas. Diabetes research clinical practice, 2019, 157: 107843.

[2] $\mathrm{Xu} \mathrm{Y,} \mathrm{Wang} \mathrm{L}, \mathrm{He}$ J, et al. Prevalence and control of diabetes in Chinese adults. JAMA, 2013, 310 (9): 948-959.

[3] Wang L, Gao P, Zhang M, et al. Prevalence and Ethnic Pattern of Diabetes and Prediabetes in China in 2013. JAMA, 2017, 317 (24): 2515-2523.

[4] YANG WJSSV. Epidemiology and trends in diabetes in China. 2018, 48 (8): 812-819.

[5] Yong L. Epidemiology and research advances in diabetes mellitus in China. Journal of Chongqing Medical University, 2015, 40 (07): 1042-1045.

[6] Mellitus DJDc. Diagnosis and classification of diabetes mellitus. 2005, 28 (S37): S5-S10.

[7] He Haiyan L, Lili, Yu Pingping. Hazard, prevention and management of needle stick injuries among health care workers J Chinese Journal of Infection Control, 2017, 16 (6): 582-586.

[8] Dong Y, Li F, Li J, et al. Multicenter cross-sectional study on the reporting status and influencing factors of needlestick injuries caused by insulin injection devices among nurses in Peking, China. Am J Infect Control, 2020, 48 (7): 805-809.

[9] Costigliola V, Frid A, Letondeur C, et al. Needlestick injuries in European nurses in diabetes. Diabetes Metab, 2012, 38 Suppl 1: S9-14.

[10] Mannocci A, De Carli G, Di Bari V, et al. How Much do Needlestick Injuries Cost? A Systematic Review of the Economic Evaluations of Needlestick and Sharps Injuries Among Healthcare Personnel. Infect Control Hosp Epidemiol, 2016, 37 (6): 635-646.

[11] Kunishima H, Yoshida E, Caputo J, et al. Estimating the national cost burden of in-hospital needlestick injuries among healthcare workers in Japan. PLoS One, 2019, 14 (11): $\mathrm{e} 0224142$.
[12] Occupational Safety and Health Administration, OSHA: Washington DC, USA. Facts about hospital worker safety. 2013.

[13] Razavi-Shearer D, Gamkrelidze I, Nguyen MH, et al. Global prevalence, treatment, and prevention of hepatitis $\mathrm{B}$ virus infection in 2016: a modelling study. 2018, 3 (6): 383-403.

[14] Fuzhen ZGMNZHSXYZW. Incidence by age and region of hepatitis B reported in China from 2005 to 2016 CHINESE JOU R NAL OF VACCINES AND IMMUNIZATION 2018, 24 (02): 121-126.

[15] Fettig J, Swaminathan M, Murrill CS, et al. Global epidemiology of HIV. 2014, 28 (3): 323-337.

[16] Zhao F, Zhang M, Xuan J, et al. Burden of insulin injection-related needlestick injuries in mainland China-prevalence, incidence, and healthcare costs. Int J Nurs Stud, 2019, 97: 78-83.

[17] Gao X, Hu B, Suo Y, et al. A large-scale survey on sharp injuries among hospital-based healthcare workers in China. Sci Rep, 2017, 7: 42620.

[18] Huang SL, Lu Q, Fan SH, et al. Sharp instrument injuries among hospital healthcare workers in mainland China: a cross-sectional study. BMJ Open, 2017, 7 (9): e017761.

[19] Lee JM, Botteman MF, Nicklasson L, et al. Needlestick injury in acute care nurses caring for patients with diabetes mellitus: a retrospective study. Curr Med Res Opin, 2005, 21 (5): 741-747.

[20] Strauss K. WISE recommendations to ensure the safety of injections in diabetes. Diabetes Metab, 2012, 38 Suppl 1: S2-8.

[21] Mondy K, Overton ET, Grubb J, et al. Metabolic syndrome in HIV-infected patients from an urban, midwestern US outpatient population. Clin Infect Dis, 2007, 44 (5): 726-734.

[22] $\mathrm{Lu} \mathrm{J}, \mathrm{Hou} \mathrm{X}, \mathrm{Tu} \mathrm{H}$, et al. Chronic hepatitis B virus infection status is more prevalent in patients with type 2 diabetes. J Diabetes Investig, 2017, 8 (4): 619-625.

[23] Fabiani S, Fallahi P, Ferrari SM, et al. Hepatitis C virus infection and development of type 2 diabetes mellitus: Systematic review and meta-analysis of the literature. Rev Endocr Metab Disord, 2018, 19 (4): 405-420.

[24] Tarigan LH, Cifuentes M, Quinn M, et al. Prevention of needle-stick injuries in healthcare facilities: a meta-analysis. Infect Control Hosp Epidemiol, 2015, 36 (7): 823-829.

[25] Zhang X, Chen Y, Li Y, et al. Needlestick and Sharps Injuries Among Nursing Students in Nanjing, China. Workplace Health Saf, 2018, 66 (6): 276-284.

[26] Cho E, Lee H, Choi M, et al. Factors associated with needlestick and sharp injuries among hospital nurses: a cross-sectional questionnaire survey. Int J Nurs Stud, 2013, 50 (8): 1025-1032.

[27] Motaarefi H, Mahmoudi H, Mohammadi E, et al. Factors Associated with Needlestick Injuries in Health Care Occupations: A Systematic Review. J Clin Diagn Res, 2016, 10 (8): IE01-IE04.

[28] Ruihua J, Jie Z, Yan WJCNR. Development status of diabetes nurse specialists in China. 2010, 27.

[29] Hamric AB, Hanson CM, Tracy MF, et al. Advanced Practice Nursing-E-Book: An Integrative Approach: Elsevier Health Sciences, 2013. 
[30] ZHAO Meicun DM, JIANG Feng, HUANG Jin. Training and management of Diabetes Specialist Nurses and its consideration and countermeasures. Chinese Nursing Management, 2019, 19 (6): 905-909.

[31] Sriram S. Study of needle stick injuries among healthcare providers: Evidence from a teaching hospital in India. J Family Med Prim Care, 2019, 8 (2): 599-603.

[32] Mischke C, Verbeek JH, Saarto A, et al. Gloves, extra gloves or special types of gloves for preventing percutaneous exposure injuries in healthcare personnel. 2014, (3).

[33] Smith DR, Choe M-A, Jeong JS, et al. Epidemiology of needlestick and sharps injuries among professional Korean nurses. 2006, 22 (6): 359-366.

[34] Lu L, Tong W, Gu L, et al. The current hepatitis C virus prevalence in China may have resulted mainly from an officially encouraged plasma campaign in the 1990s: a coalescence inference with genetic sequences. 2013, 87 (22): 12041-12050.

[35] Wang Li yan QQ, Ding Zhengwei, CaiChang. Current situation of HIV epidemic in China. Chin J A I D S STD, 2017, 23 (04): 330-333.

[36] Fukuda H, Yamanaka N. Reducing needlestick injuries through safety-engineered devices: results of a Japanese multi-centre study. J Hosp Infect, 2016, 92 (2): 147-153.
[37] Chambers A, Mustard CA, Etches JJBhsr. Trends in needlestick injury incidence following regulatory change in Ontario, Canada (2004-2012): an observational study. 2015, 15 (1): $1-6$.

[38] Mohammadnejad E, Nemati Dopolani FJJoN, Sciences M. Risk factors of needle stick and sharp injuries among health care workers. 2015, 2 (1): 34-39.

[39] Ishak A, Haque M, Sadhra SJOM. Needlestick injuries among Malaysian healthcare workers. 2019, 69 (2): 99-105.

[40] Organization WH. WHO guideline on the use of safety-engineered syringes for intramuscular, intradermal and subcutaneous injections in health-care settings. In: World Health Organization, 2015.

[41] Joukar F, Mansour-Ghanaei F, Naghipour M, et al. Needlestick Injuries among Healthcare Workers: Why They Do Not Report their Incidence? Iran J Nurs Midwifery Res, 2018, 23 (5): 382-387.

[42] Sabermoghaddam M, Sarbaz M, Lashkardoost H, et al. Incidence of occupational exposure to blood and body fluids and measures taken by health care workers before and after exposure in regional hospitals of a developing country: A multicenter study. 2015, 43 (10): 1137-1138. 